\title{
WEB GEOMETRY
}

\author{
BY SHIING-SHEN CHERN ${ }^{1}$
}

Introduction. Poincaré published two papers on surfaces of translation [10, 11]. ${ }^{2}$ They were among his lesser known papers. In the following pages I wish to show that the subject he touched is an exciting one and deserves further investigation.

1. Lie's theorem on surfaces of double translation and its developments. A surface $M$ of translation in $R^{3}$ is defined by the parametric equations

$$
x^{\lambda}=f^{\lambda}(u)+g^{\lambda}(v), \quad 1 \leqslant \lambda \leqslant 3,
$$

where $x^{\lambda}$ are the coordinates in $R^{3}$ and $f^{\lambda}, g^{\lambda}$ are arbitrary smooth functions. It is immediately seen that the tangent lines to the $u$-curves (respectively the $v$ curves) are independent of $v$ (resp. $u$ ) and define a curve $C_{u}$ (resp. $C_{v}$ ) in the plane at infinity.

$M$ is called a surface of double translation if it is a surface of translation in a second way, i.e., given also by the equations

$$
x^{\lambda}=h^{\lambda}(s)+k^{\lambda}(t), \quad 1 \leqslant \lambda \leqslant 3,
$$

such that exactly two of the equations

$$
f^{\lambda}(u)+g^{\lambda}(v)-h^{\lambda}(s)-k^{\lambda}(t)=0, \quad 1 \leqslant \lambda \leqslant 3,
$$

are independent. In 1882 Sophus Lie proved the remarkable theorem [7]:

If $M$ is a surface of double translation in $R^{3}$, the four curves $C_{u}, C_{v}, C_{s}, C_{t}$ in the plane at infinity defined by the tangent lines to the four families of parametric curves belong to the same algebraic curve of degree four.

The theorem means that the solutions of the functional equations (3) on a surface arise from an algebraic structure. Lie's proof makes use of the integrability conditions of over-determined systems of partial differential equations. In fact, from (1) we have

$$
\partial^{2} x^{\lambda} / \partial u \partial v=0
$$

which means that the parametric curves form a conjugate net, i.e., their tangent directions separate harmonically the asymptotic directions at each point. If the surface $M$ is given in the nonparametric form

$$
z=z(x, y)
$$

Received by the editors November 12, 1980.

1980 Mathematics Subject Classification. Primary 53A60, 14D25.

${ }^{1}$ Work done under partial support of NSF grant MC577-23579.

${ }^{2}$ Numbers in brackets refer to the Bibliography at the end of the paper.

(C) 1982 American Mathematical Society $0002-9904 / 82 / 0000-0243 / \$ 03.00$ 
this condition is expressed by an equation

$$
R(p, q) r+Q(p, q) s+T(p, q) t=0
$$

where

$$
p=z_{x}, \quad q=z_{y}, \quad r=z_{x x}, \quad s=z_{x y}, \quad t=z_{y y} .
$$

A surface $M$ of double translation satisfies, besides (6), another equation

$$
R^{\prime}(p, q) r+Q^{\prime}(p, q) s+T^{\prime}(p, q) t=0 .
$$

An investigation of the integrability conditions of the system of equations (6), $\left(6^{\prime}\right)$ involves long and tedious calculations. In particular, the fourth-order partial derivatives of $z$ come into play. The work was a true tour de force, but Lie reached his goal.

Poincaré was quick to recognize the importance of Lie's work, and to observe its relation with the theory of abelian functions. In $[10,11]$ he gave two proofs of Lie's theorem based on abelian functions and algebraic geometry rather than on partial differential equations. ${ }^{3}$ Although the proofs are perhaps not complete, Poincaré introduced fresh ideas and new viewpoints. As a consequence of Poincarés work it follows that a surface of double translation can be defined by equating a theta function to zero. As a result the surface

$$
x_{1} x_{2} x_{3}=a_{1} x_{1}+a_{2} x_{2}+a_{3} x_{3},
$$

where the $a$ 's are constants, is a surface of double translation. The best proof of Lie's theorem was given by Darboux, using the theory of residues [5].

It should be remarked that Lie started his program on surfaces of translation through his work on minimal surfaces. It was known to Monge that an analytic minimal surface is a surface of translation (1) with parametric curves which are minimal or isotropic curves.

Lie proceeded to study the high-dimensional case. A translation manifold in $R^{n+1}$ is the hypersurface defined by the parametric equations

$$
x^{\lambda}=\sum_{1<\alpha<n} f^{\lambda}\left(u_{\alpha}\right), \quad 1 \leqslant \lambda \leqslant n+1,
$$

where $x^{\lambda}$ are the coordinates in $R^{n+1}$ and the $f^{\prime}$ 's are smooth functions in the respective variables. Lie tried to determine all hypersurfaces of double translation and settled the case $n=3$ in a long paper [8]. In the same paper he promised to return to the general case. He had several posthumous papers on the subject, without bringing the problem to a satisfactory conclusion [9]. The high-dimensional case was also considered by Poincaré. It was W. Wirtinger who in 1938 completely solved the problem, using Chow coordinates for projective varieties [14]. We will show below that web geometry offers a broader setting where the subject can be integrated.

\footnotetext{
${ }^{3}$ Lie was unhappy with Poincaré's intrusion: He said: "Unfortunately the distinguished author (i.e., Poincaré), whose achievements in other fields were recognized by nobody more than myself, failed to understand my investigations. I can only say that his works on translation surfaces and translation manifolds deal with results which are entirely special cases of my general theorems." (Ges. Abh, Bd II, Teil II, 527)
} 
The conclusion that the functional equations imply an algebraic structure is a powerful one. This was utilized by B. St. Donat in 1975 to give a new proof of Torelli's theorem that a compact Riemann surface is determined up to isomorphism by its period matrix (or more exactly, by its polarized Jacobian variety) [12].

2. Web geometry of Blaschke-Bol [2]. Web geometry had its debut in 1926-27 on the beaches of Italy when W. Blaschke and G. Thomsen realized that the configuration of three foliations of the plane by curves, has local invariants. The distinguished geometric figure in this case is the hexagon (Figure 1). When all such hexagons are closed, for any point $O$ and any neighboring point $P$ on the first curve through $O$, the web is called hexagonal. Thomsen proved that a hexagonal web is locally homeomorphic to three families of parallel lines.

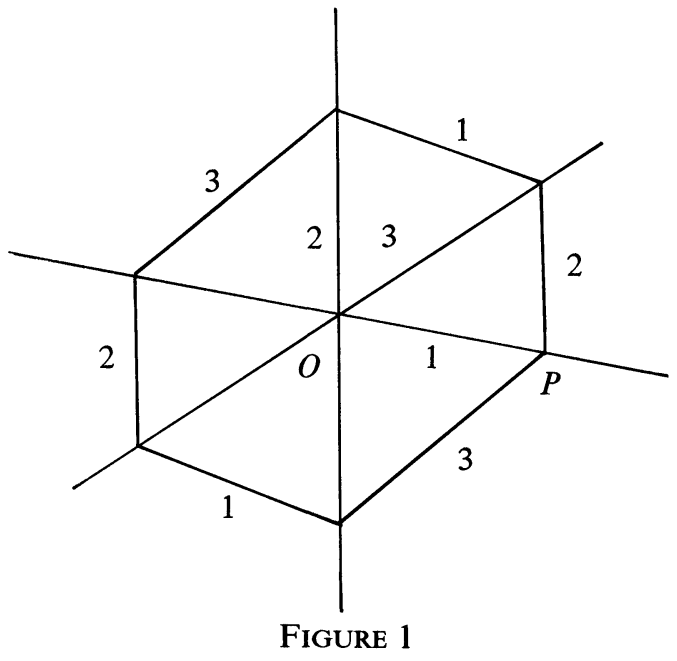

The relation of this subject to algebraic geometry is immediate. Before Blaschke and Thomsen started their work on web geometry, Graf and Sauer proved in 1924 a theorem which, in web geometry language, can be stated as follows: If the curves of a hexagonal web are straight lines, they are the tangent lines of an algebraic curve of class three.

Generally, a $d$-web in the plane is defined by $d$ foliations in a neighborhood of the plane by curves such that through every point the $d$ leaves have mutually distinct tangents. If $x, y$ are the coordinates in the plane, a $d$-web can be defined by

$$
u_{i}(x, y)=\text { const, } \quad 1 \leqslant i \leqslant d .
$$

We shall assume that the functions $u_{i}(x, y)$ are smooth and satisfying the condition $\operatorname{grad} u_{i} \neq 0$.

An equation of the form

$$
\sum_{1<i<d} f_{i}\left(u_{i}\right) d u_{i}=0
$$


is called an abelian equation. The maximum number of linearly independent abelian equations, is called the rank of the web, to be denoted by $\pi$. It can be proved that

$$
\pi \leqslant \frac{1}{2}(d-1)(d-2) .
$$

We see easily that for $d=3$, the rank one webs are exactly the hexagonal webs. For $d=4$, we have $\pi \leqslant 3$, and the 4-webs of rank 3 satisfy 3 linearly independent abelian equations

$$
\sum f_{i}^{\lambda}\left(u_{i}\right) d u_{i}=0, \quad 1 \leqslant \lambda \leqslant 3 .
$$

By setting

$$
x^{\lambda}=\int f_{1}^{\lambda}\left(u_{1}\right) d u_{1}+\int f_{2}^{\lambda}\left(u_{2}\right)=-\int f_{3}^{\lambda}\left(u_{3}\right) d u_{3}-\int f_{4}^{\lambda}\left(u_{4}\right) d u_{4}, \quad 1 \leqslant \lambda \leqslant 3,
$$

we get in $R^{3}$ a surface of double translation. Lie's theorem can be interpreted as saying that a 4-web of rank 3 , is locally equivalent to one consisting of straight lines; the latter must then be the tangent lines of a curve of class 4 .

The deep question is whether any $d$-web of maximum rank $(d-1)(d-2) / 2$ is locally equivalent to one all whose leaves are straight lines. Bol gave an example showing that this is not always the case for a 5-web of rank 6. Bol's example consists of four pencils of lines, no three of whose vertices are collinear, with the fifth family consisting of the conics through the four vertices. Of the six abelian equations one involves the transcendental function which is Euler's dilogarithm. The latter has played a role in several recent mathematical investigations, such as the volume of a simplex in an odd-dimensional noneuclidean space, the Pontrjagin number of a combinatorial 4-dimensional manifold and, more generally, in algebraic $K$-theory. A primary reason must be the fact that it satisfies an abelian equation. For a recent work cf. [15].

3. High-dimensional webs. A $d$-web of dimension $N-k$ in $R^{N}$ consists of $d$ foliations of a neighborhood $U$ of $R^{N}$ by submanifolds of dimension $N-k$; $k$ is called the codimension of the web. As an example consider an algebraic variety $V$ of dimension $k$ and degree $d$ in a projective space $P^{m}$ of dimension $m$. A linear subspace $P^{m-k}$ of dimension $m-k$ meets $V$ in $d$ points through each of which pass $\infty^{k(m-k)} P^{m-k}$ 's. This gives in the Grassmann manifold $G(m-k, m)$ of all $P^{m-k}$ 's in $P^{m} d$ foliations of dimension $k(m-k)$. Since

$$
\operatorname{dim} G(m-k, m)=k(m-k+1),
$$

the variety $V$ gives rise to $d$ foliations of codimension $k$ in $G(m-k, m)$, which is locally $R^{k n}, n=m-k+1$. In order to keep algebraic geometry in sight, we will consider on the basis of this example, only webs of codimension $k$ in $R^{N}$, with $N=k n$. Even so the subject is a wide generalization of the geometry of projective algebraic varieties. Just as intrinsic algebraic varieties are generalized to Kähler manifolds and complex manifolds, such a generalization to web geometry seems justifiable. 
The tangent spaces to the $d$ leaves through a point $x \in R^{k n}$ give $d$ linear subspaces of codimension $k$ in the tangent space $T_{x}$ to $R^{k n}$ at $x$ or, what is the same, $d$ linear subspaces $\Omega_{i}$ of dimension $k$ in the cotangent space $T_{x}^{*}$. We will suppose that they are in general position. For $k=1$ the meaning of this is clear: no $k n=n$ of the $d$ lines in $T_{x}^{*}$ lie in a hyperplane of $T_{x}^{*}$. For $k>1$ the right notion has to be introduced. We gave one in [4], again based on the example of projective varieties.

Analytically suppose the $i$ th foliation of the web be defined by the equations

$$
u_{i 1}(x)=\text { const }, \quad, \ldots, \quad u_{i k}(x)=\text { const, } \quad 1 \leqslant i \leqslant d, \quad x \in R^{k n} .
$$

We will suppose the $u$ 's to be smooth functions satisfying

$$
\Omega_{i}(x)=d u_{i 1} \wedge \cdots \wedge d u_{i k} \neq 0 .
$$

For a fixed $i$ the functions $u_{i 1}, \ldots, u_{i k}$ are defined up to a diffeomorphism and $\Omega_{i}(x)$ is defined up to a factor. The notation is so chosen that it defines the linear space $\Omega_{i} \subset T_{x}^{*}$ introduced above; we will call it the $i$ th web normal.

An abelian equation is an equation of the form

$$
\sum_{1<i<d} f_{i}\left(u_{i 1}, \ldots, u_{i k}\right) \Omega_{i}=0 .
$$

The maximum number of the linearly independent abelian equations is called the rank of the web. In [4] we proved that the rank has an upper bound $\pi(d, n, k)$, which depends only on $d, n, k$. This bound is sharp. In particular, for $k=1$ we have

$$
\pi(d, n) \underset{\text { def }}{=} \pi(d, n, 1)=(1 / 2(n-1))\{(d-1)(d-n)+s(n-s-1)\}
$$

where $s$ is defined by

$$
s \equiv-d+1 \bmod n-1, \quad 0 \leqslant s \leqslant n-2 .
$$

This number $\pi(d, n)$ has an important meaning in algebraic geometry. In fact, Castelnuovo proved that $\pi(d, n)$ is the maximum genus of an algebraic curve of degree $d$ in $P^{n}$, which does not belong to a hyperplane $P^{n-1}$. By taking such a curve $C^{*}$ in the dual space $P^{* n}$, we have through each point of $P^{n} d$ hyperplanes belonging to $C^{*}$ and it follows from Abel's theorem that the $d$-web so constructed is of rank $\pi(d, n)$. In relating web geometry to algebraic geometry it should be remarked that our web geometry is over real numbers while the corresponding notions of algebraic geometry refer to the complex field. The transition is not immediate, but it is possible owing mainly to the fact that we are dealing with local properties in web geometry.

It is clear that an important problem is to determine the $d$-webs of codimension 1 and maximum rank $\pi(d, n)$ in $R^{n}$. If the leaves are all hyperplanes, the answer is given by the following converse to Abel's [6] theorem (generalization of theorems of Graf-Sauer and Lie): 
Consider a d-web of codimension 1 in a neighborhood of $R^{n}$ whose leaves are hyperplanes such that an abelian equation

$$
\sum_{1<i<d} f_{i}\left(u_{i}\right) d u_{i}=0
$$

holds, with $f_{i}\left(u_{i}\right) \neq 0$. Then the leaves belong to an algebraic curve of degree $d$ in the dual projective space.

For this theorem it is sufficient to have one abelian equation. The crucial question is thus the linearization problem: Is a $d$-web of codimension one and rank $\pi(d, n)$ in $R^{n}$ linearizable, i.e., is it locally equivalent to one having hyperplanes as leaves? Bol's example in $\$ 2$ shows that the answer is no for $n=2, d=5$.

Consider next the case $n \geqslant 3$. For $n+1 \leqslant d \leqslant 2 n-1$ we have $\pi(d, n)=$ $d-n$, and there are simple examples of nonlinearizable $d$-webs of rank $d-n$, which depend on arbitrary functions. Lie's case of hypersurfaces of double translation corresponds to $d=2 n$. In this case we have $\pi(2 n, n)=n$ +1 . For a $2 n$-web of rank $n+1$ the abelian equations

$$
\sum f_{i}^{\lambda}\left(u_{i}\right) d u_{i}=0, \quad 1 \leqslant \lambda \leqslant \pi=n+1
$$

can be written

$$
\sum \int_{1<i \leqslant n} f_{i}^{\lambda}\left(u_{i}\right) d u_{i}=-\sum \int_{n+1 \leqslant i<2 n} f_{i}^{\lambda}\left(u_{i}\right) d u_{i}, \quad 1 \leqslant \lambda \leqslant n+1 .
$$

As a generalization of (14) these common expressions can be regarded as the coordinates in $R^{n+1}$ of a hypersurface of double translation.

An essential step in the proof of the Lie-Wirtinger theorem on hypersurfaces of double translation is to show that a $2 n$-web in $R^{n}$ of codimension one and rank $n+1$ is linearizable, from which the theorem follows from the converse to Abel's theorem. In this particular case the linearization follows by a simple argument, using an idea of Poincaré. It goes as follows: For $x \in U \subset R^{n}$ let $Z_{i}(x)$ be the point in a projective space of dimension $\pi-1$, whose homogeneous coordinates are $\left[f_{i}^{1}\left(u_{i}\right), \ldots, f_{i}^{\pi}\left(u_{i}\right)\right]$. The mapping which sends $x \in U \subset R^{n}$ to the space $\left\{Z_{1}, \ldots, Z_{d}\right\} \subset P^{\pi-1}$ spanned by the $Z_{i}$ 's is called Poincaré's mapping. If $u_{1}, \ldots, u_{n}$ are regarded as a local coordinate system in $U$, we have, from (21),

$$
f_{1}^{\lambda}\left(u_{1}\right)+\sum_{n+1 \leqslant i \leqslant d} f_{i}^{\lambda}\left(u_{i}\right) \frac{\partial u_{i}}{\partial u_{1}}=0, \quad 1 \leqslant \lambda \leqslant \pi .
$$

It follows that $Z_{1}$ is a linear combination of $Z_{n+1}, \ldots, Z_{d}$. Since a similar equation holds for any $Z_{i}$ (instead of $Z_{1}$ ), we have

$$
\left\{Z_{1}, \ldots, Z_{d}\right\}=d-n-1 \text {. }
$$

In the case $d=2 n, \pi=n+1$, the Poincare mapping maps the points of $U$ into hyperplanes of $P^{n}$, such that the $i$ th leaf goes to the point $Z_{i}$. The Poincaré mapping followed by duality in $P^{n}$ maps the points of $U$ into the points of the dual space $P^{* n}$ such that all the leaves of the web go to 
hyperplanes. This proves the linearization, and the Lie-Wirtinger theorem follows.

For $n=3 \mathrm{Bol}$ proved the remarkable theorem: In $R^{3}$ a $d$-web of codimension 1 and rank $\pi(d, 3), d \geqslant 6$, is linearizable.

Griffiths and I tried to generalize Bol's theorem to $R^{n}$ [3]. So far we have only succeeded to prove the theorem under the additional condition of normality. The exact statement is: In $R^{n}$ a normal $d$-web of codimension 1 and rank $\pi(d, n), d \geqslant 2 n$, is linearizable. For the definition of normality we refer to [3].

In recent years works on web geometry have also been done by $\mathbf{M}$. A. Akivis and V. Goldberg in the Soviet Union. It is not clear to me how much these works are related to those of Poincaré. The reader is referred to [1] for further information.

Tschebotarow generalized Lie's idea to the study of surfaces which admit imprimitive systems relative to an arbitrary Lie group (instead of the group of translations) [13]. To my knowledge this generalization has not been further pursued.

4. Unsolved problems. In the following I wish to list a few most immediate unsolved problems:

1. Determine all $d$-webs of curves in the plane having maximum rank $\frac{1}{2}(d-1)(d-2), d \geqslant 5$.

2. Is the above linearization theorem in $R^{n}, n \geqslant 4$, true without normality?

3 (Griffiths'). The hexagonal condition is meaningful for a 3 -web of dimension $k$ in $R^{2 k}$. The construction described in the beginning of $\$ 3$, with $m=k+1$, so that $n=2$, defines from a cubic hypersurface in $P^{k+1}$ a 3-web of dimension $k$ in $R^{2 k}$. It can be shown that such a web is hexagonal. Is the converse true, i.e., does every hexagonal 3-web of dimension $k$ in $R^{2 k}$ arise from such a construction (up to a local diffeomorphism)?

ADDED DURING PROOF. I am indebted to V. Goldberg that the answer to this question is "no".

4. Lie's treatment makes heavy use of overdetermined systems of partial differential equations. Give a proof of the Lie-Wirtinger theorem by PDE.

\section{REFERENCES}

1. M. A. Akivis, Webs and almost Grassmann structures, Soviet Math. Dokl. 21 (1980), no. 3. Further references to works by V. Goldberg and other Soviet mathematicians on the subject can be found in this paper.

2. W. Blaschke and G. Bol, Geometrie der Gewebe, Springer, Berlin, 1938.

3. S. S. Chern and P. A. Griffiths, Abel's theorem and webs, Jahresberichte der deut. Math. Ver. 80 (1978), 13-110; also, Corrections and Addenda, same Journal, 83 (1981), 78-83.

4. An inequality for the rank of $a$ web and webs of maximum rank, Ann. Scuola Norm. Pisa, Serie IV, 5 (1978), 539-557.

5. G. Darboux, Théorie des surfaces, t.1, (1914), 151-161.

6. P. A. Griffiths, Variations on a theorem of Abel, Invent. Math. 35 (1976), 321-390.

7. S. Lie, Bestimmung aller Flächen, die in mehrfacher Weise durch Translationsbewegung einer Kurve erzeugt werden, Arch. für Math. Bd. 7, Heft 2 (1882), 155-176; also Ges. Abhandlungen, Bd. 1, Abt 1, 450-467. 
8. Das Abelsche Theorem und die Translationsmannigfaltigkeiten, Leipziger Berichte 1897, pp. 181-248; also Ges. Abhandlungen, Bd. II, Teil II, paper XIV, pp. 580-639.

9. Ges. Abhandlungen, Bd. 7.

10. H. Poincaré, Fonctions abeliennes, J. Math. Pures Appl., Serie 5, t.1, (1895), 219-314; also Oeuvres t.IV, pp. 384-472, in particular p. 430.

11. _ Sur les surfaces de translation et les fonctions abeliennes, Bull. Soc. Math. France 29 (1901), 61-86; also Oeuvres t.VI, pp. 13-37.

12. B. Saint-Donat, Variétés de translation et théorème de Torelli, Comptes Rendus Paris 280 (1975), 1611-1612.

13. N. Tschebotarow, Über Flächen welche Imprimitivitätssysteme in Bezug auf eine gegebene Kontinuirliche Transformationsgruppe enthalten, Recueil Math. (Sbornik) 34 (1927), 149-204.

14. W. Wirtinger, Lies Translationsmannigfaltigkeiten und Abelsche Integrale, Monatshefte Math. Phys. 46 (1938), 384-431.

15. David B.. Damiano, Webs, abelian equations, and characteristic classes, Thesis, Brown University, 1980.

16. John B. Little, Translation manifolds and the converse of Abel's theorem, Thesis, Yale University, 1980.

Department of Mathematics, University of California, Berkeley, California 94720 